\title{
Research on the influence of electric vehicle driven system on vehicle shimmy based on numerical calculation
}

\author{
Xiaogao $\mathrm{Li}^{1,2}$, Ning Zhang ${ }^{1}$, and Nan Chen ${ }^{1, *}$ \\ ${ }^{1}$ School of Mechanical Engineering, Southeast University, Nanjing 211189, China \\ ${ }^{2}$ School of Mechanical and Electronic Engineering, Jingdezhen Ceramic Institute, Jingdezhen 333403, \\ China
}

\begin{abstract}
A 6 degrees of freedom shimmy model for four in-wheel motors independent drive electric vehicle with independent front suspension is established, and numerical analysis and simulation are used to study the dynamic response of vehicle shimmy. The influence of electric vehicle driving system on shimmy is studied by comparing with fuelengined vehicle, and the influence of vehicle structural parameters such as the caster angle, the inclination angle of front suspensions and the centre of gravity of vehicle on shimmy are studied too. It shows that as the in-wheel motor in drive system increases the weight of wheel, the amplitude of each degree of freedom in electric vehicle are larger than in fuel-engined vehicle when vehicle shimmies. The influence of the caster angle and the centre of gravity of vehicle on vehicle shimmy is obvious, but the inclination angle of front suspension have little influence.
\end{abstract}

\section{Introduction}

The new energy vehicle, represented by electric vehicle (EV) whose core is electric driven system, has become one of the commanding points of vehicle research and development and even industrial competition all over the world. Structure with independent suspension and driven by in-wheel motors or wheel-side motors independently is considered to be the most effective configuration of EV with the essential advantages of electric drive, and has attracted the attention from the global EV industry. ${ }^{[1]-[3]}$ Vehicle shimmy is a continuous vibration of the steering wheels around they respective kingpin when vehicle travels on flat road. As early as the $1930 \mathrm{~s}$, some researchers began to study the problem of vehicle shimmy. ${ }^{[4]}$ In recent decades, researchers have carried out extensive research on the problem of vehicle shimmy, and a series achievement have been made..$^{[5]-[8]}$

But at present, the object of research on the problem of vehicle shimmy is mainly traditional fuel-engined vehicle (FEV), and the problem of front wheels shimmy in EV has hardly been raised specially. Because of the introduction of driving motors and power battery, the structures of EV and FEV are extremely different. Thus the shimmy of EV and FEV are not identical. The shimmy problem of four in-wheel motors independent drive

\footnotetext{
* Corresponding author: nchen@seu.edu.cn
} 
(FIMID) EV and the influence of vehicle parameters on EV shimmy will be studied in this paper.

\section{Electric vehicle shimmy model}

\subsection{Structure of electric vehicle}

The diagram of the structure of FIMID EV, where four in-wheel motors driven and controlled by the driver are mounted in its wheels, and engine is replaced by power battery to provide power for vehicle, is shown in Figure 1. In-wheel motors increase the weight of wheels and the unsprung mass of vehicle, thus the center of gravity (COG) of whole vehicle is lowered, and its safety is improved. Power battery are usually lithium battery or lead acid battery. Because power batteries are usually placed in the middle of vehicle, or placed in the rear, the position of the COG of whole EV is changed.

\subsection{Mechanical model of electric vehicle shimmy}

The mechanical model of FIMID EV front wheels shimmy is shown in Figure 2, where $(a)$ is the top view, $(b)$ is the rear view. The mechanical model of FIMID EV front wheels shimmy has 6 degrees of freedom (DOF): the swing of front-left and front-right wheels around their respective kingpin $\theta_{1}$ and $\theta_{2}$, namely the front wheels shimmy, the lateral swing of front-left and front-right wheel axes around $x$ axis $\varphi_{1}$ and $\varphi_{2}$, the swing of pitman arm in steering system around its rotating center $\theta_{3}$, the lateral sway of whole vehicle along $y$ axis.

\subsection{Mathematical model of electric vehicle shimmy}

Based on the mechanical model as shown in Figure 2, and using Lagrange's equation, its mathematical model can be obtained. In order to reduce the length of this paper, modeling process is omitted, and the mathematical model of FIMID EV front wheels shimmy can be represented by equation (1).

$$
\left\{\begin{array}{l}
\left(J_{\alpha}+J_{d} \beta^{2} \theta_{1}^{2}\right) \ddot{\theta}_{1}-\left[J_{\gamma}+\left(J_{d}-J_{0}\right) \beta \theta_{1}\right] \ddot{\varphi}_{1}+\left(k_{1} l_{d}^{2}+k_{b} l_{b}^{2} \gamma^{2}+k_{y} R^{2} \gamma^{2}\right) \theta_{1}+\left(c_{e}+c_{1} l_{d}^{2}\right) \dot{\theta}_{1}-k_{1} l_{d} l_{g} \theta_{3} \\
-c_{1} l_{d} l_{g} \dot{\theta}_{3}-\left(k_{y} R^{2} \gamma+k_{b} l_{b} l_{f} \gamma-k_{b} l_{b} l_{f} f\right) \varphi_{1}+J_{0} \frac{v}{R} \dot{\varphi}_{1}+J_{d} \beta^{2} \theta_{1} \dot{\theta}_{1}^{2}-J_{0} \theta_{1} \dot{\varphi}_{1}^{2}=-F_{Y 1}(R \gamma+e) \\
\left(J_{\alpha}+J_{d} \beta^{2} \theta_{2}^{2}\right) \ddot{\theta}_{2}-\left[J_{\gamma}-\left(J_{d}-J_{0}\right) \beta \theta_{2}\right] \ddot{\varphi}_{2}+\left(k_{2} l_{d}^{2}+k_{b} l_{b}^{2} \gamma^{2}+k_{y} R^{2} \gamma^{2}\right) \theta_{2}+\left(c_{e}+c_{2} l_{d}^{2}\right) \dot{\theta}_{2}-k_{2} l_{d} l_{g} \theta_{3} \\
-c_{2} l_{d} l_{g} \dot{\theta}_{3}-\left(k_{y} R^{2} \gamma+k_{b} l_{b} l_{f} \gamma-k_{b} l_{b} l_{f} f\right) \varphi_{2}+J_{0} \frac{v_{2}}{R} \dot{\varphi}_{2}+J_{d} \beta^{2} \theta_{2} \dot{\theta}_{2}^{2}-J_{0} \theta_{2} \dot{\varphi}_{2}^{2}=-F_{Y 2}(R \gamma+e) \\
J_{p} \ddot{\theta}_{3}-k_{1} l_{d} l_{g} \theta_{1}-c_{1} l_{d} l_{g} \dot{\theta}_{1}-k_{2} l_{d} l_{g} \theta_{2}-c_{2} l_{d} l_{g} \dot{\theta}_{2}+\left(k_{1} l_{g}^{2}+k_{2} l_{g}^{2}+k_{3}\right) \theta_{3}+\left(c_{1} l_{g}^{2}+c_{2} l_{g}^{2}+c_{3}\right) \dot{\theta}_{3}=0 \\
\left(J_{\beta}+J_{0} \theta_{1}^{2}\right) \ddot{\varphi}_{1}-\left[J_{\gamma}+\left(J_{d}-J_{0}\right) \beta \theta_{1}\right] \ddot{\theta}_{1}-\left(k_{b} l_{f} l_{b}+k_{y} R^{2}\right) \gamma \theta_{1}-J_{0} \frac{v}{R} \dot{\theta}_{1} \\
+\left(k_{4} k_{h c}^{2} l_{a c}^{2}+k_{b} l_{f}^{2}+k_{y} R^{2}\right) \varphi_{1}+c_{4} k_{h c}^{2} l_{a c}^{2} \dot{\varphi}_{1}-\left(J_{d}-J_{0}\right) \beta \dot{\theta}_{1}^{2}+2 J_{0} \theta_{1} \dot{\theta}_{1} \dot{\varphi}_{1}=F_{Y 1} R \\
\left(J_{\beta}+J_{0} \theta_{2}^{2}\right) \ddot{\varphi}_{2}-\left[J_{\gamma}-\left(J_{d}-J_{0}\right) \beta \theta_{2}\right] \ddot{\theta}_{2}-\left(k_{b} l_{f} l_{b}+k_{y} R^{2}\right) \gamma \theta_{2}-J_{0} \frac{v}{R} \dot{\theta}_{2} \\
+\left(k_{5} k_{h c}^{2} l_{a c}^{2}+k_{b} l_{f}^{2}+k_{y} R^{2}\right) \varphi_{2}+c_{5} k_{h c}^{2} l_{a c}^{2} \dot{\varphi}_{2}+\left(J_{d}-J_{0}\right) \beta \dot{\theta}_{2}^{2}+2 J_{0} \theta_{2} \dot{\theta}_{2} \dot{\varphi}_{2}=F_{Y 2} R \\
M \dot{y}+4 k_{y} y=2\left(F_{Y 1}+F_{Y 2}\right)
\end{array}\right.
$$

In which, $v$ is the velocity of EV, $J_{0}$ and $J_{d}$ are the moments of inertia of wheel around its spin axes and diameter respectively, $J_{p}$ is the moment of inertia of pitman arm; $m_{w}$ and 
$m_{s}$ are the mass of wheel and vehicle body respectively; $k_{1}$ and $k_{2}$ are the stiffness of left and right steering tie rods, $k_{3}$ is the stiffness of pitman arm, $k_{4}$ and $k_{5}$ are the stiffness of front and rear suspensions, $c_{1}$ and $c_{2}$ are the damping of left and right steering tie rods, $c_{3}$ is the damping of pitman arm, $c_{4}, c_{5}$ are the damping of front and rear suspensions, $c_{e}$ is the equivalent damping of front wheel around kingpin, $k_{y}$ is tire lateral stiffness, $k_{b}$ is tire vertical stiffness, $f$ is the friction coefficient between tire and ground, $\gamma$ is caster angle, $e$ is pneumatic trail, $R$ is the roll radius of tire, parameters related to structural size such as $l_{a}, l_{b}$, $l_{c}, l_{d}, l_{f}, l_{g}, l_{h}$ are marked on Figure 2. $J_{\alpha}, J_{\beta}, J_{\gamma}$ can be obtained from equation (2), $l_{a c}, k_{h c}$, can be obtained from equation (3).

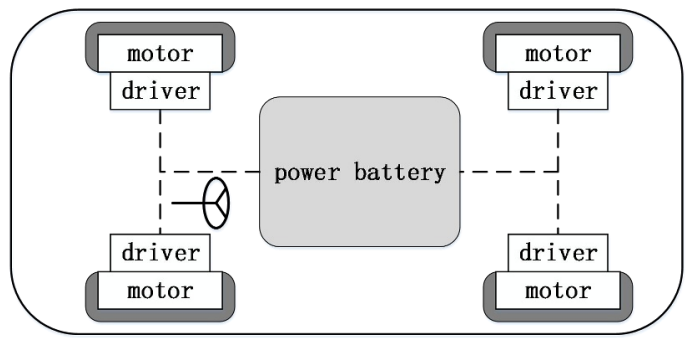

Fig. 1. Diagram of electric vehicle structure.

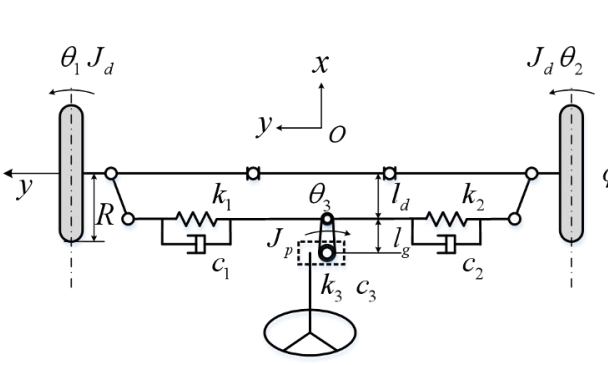

(a)

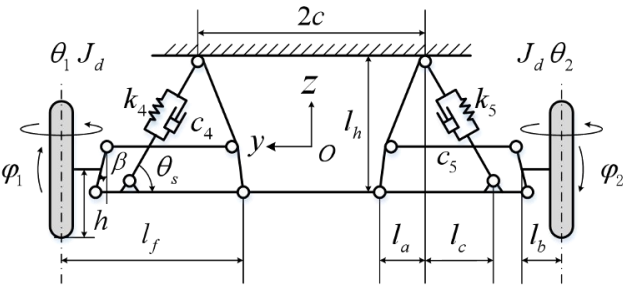

(b)

Fig. 2. Mechanical model of electric vehicle shimmy.

$$
\begin{gathered}
J_{\alpha}=J_{d}+m_{w} l_{b}^{2}+m_{w} l_{b}^{2} \gamma^{2}+J_{0} \beta^{2}, \quad J_{\beta}=J_{d}+m_{w} l_{f}{ }^{2}+J_{d} \gamma^{2}, \quad J_{\gamma}=\left(J_{d}+m_{w} l_{b} l_{f}\right) \gamma \\
l_{a c}=l_{a}+l_{c}, \quad k_{h c}=l_{h} / \sqrt{l_{h}^{2}+l_{c}{ }^{2}}
\end{gathered}
$$

$F_{Y 1}$ and $F_{Y 2}$ are the lateral forces applied on the front-right and front-left wheels of EV respectively. According to the "Magic Formula" tire model proposed by Pacejka, the lateral force acting on wheel can be given as following.

$$
F_{Y}=D \sin \{C \arctan [B \alpha(1-E)+E \arctan B \alpha]\}
$$

In which, $\alpha$ is the side-slip angle of wheel, $B$ is the stiffness factor, $C$ is the shape factor, $D$ is the peak value, $E$ is the curvature factor, and $B, C, D, E$ can be obtained from equation (5).

$$
C=a_{0}, \quad D=a_{1} F_{z}^{2}+a_{2} F_{z}, \quad B C D=a_{3} \sin \left(2 \arctan \frac{F_{z}}{a_{4}}\right) \times\left(1-a_{5}|\eta|\right), \quad E=a_{6} F_{z}+a_{7}
$$

In which, $F_{z}$ is the normal load acting on wheel, $\eta$ is the camber angle of front wheels, the parameter $a_{0} \sim a_{7}$ are constants determined for each tire.

It is assumed that EV have no lateral acceleration, the normal loads acting on the frontleft and front-right wheels $F_{Z 1}$ and $F_{Z 2}$ can be given as following.

$$
F_{z 1}=F_{z 0}-k_{b} l_{f} \varphi_{1}, \quad F_{z 2}=F_{z 0}+k_{b} l_{f} \varphi_{2}
$$


In which, $F_{Z 0}$ is the static normal load acting on the front wheels, and it can be given as following.

$$
F_{z 0}=\frac{1}{2} \frac{L_{r}}{L_{f}+L_{r}}\left(m_{s}+4 m_{w}\right) g
$$

In which, $L_{f}$ and $L_{r}$ are the distance from the COG of EV to its front axle and rear axle respectively.

According to string theory, the constraints of the side-slip angles and the shimmy angles in front wheels can be given as following.

$$
\dot{\alpha}_{1}+\frac{v}{\sigma} \alpha_{1}+\frac{v}{\sigma} \theta_{1}-\frac{a}{\sigma} \dot{\theta}_{1}=0, \quad \dot{\alpha}_{2}+\frac{v}{\sigma} \alpha_{2}+\frac{v}{\sigma} \theta_{2}-\frac{a}{\sigma} \dot{\theta}_{2}=0
$$

In which, $\sigma$ and $a$ are the relaxation length of tire and the half-length of contact area respectively.

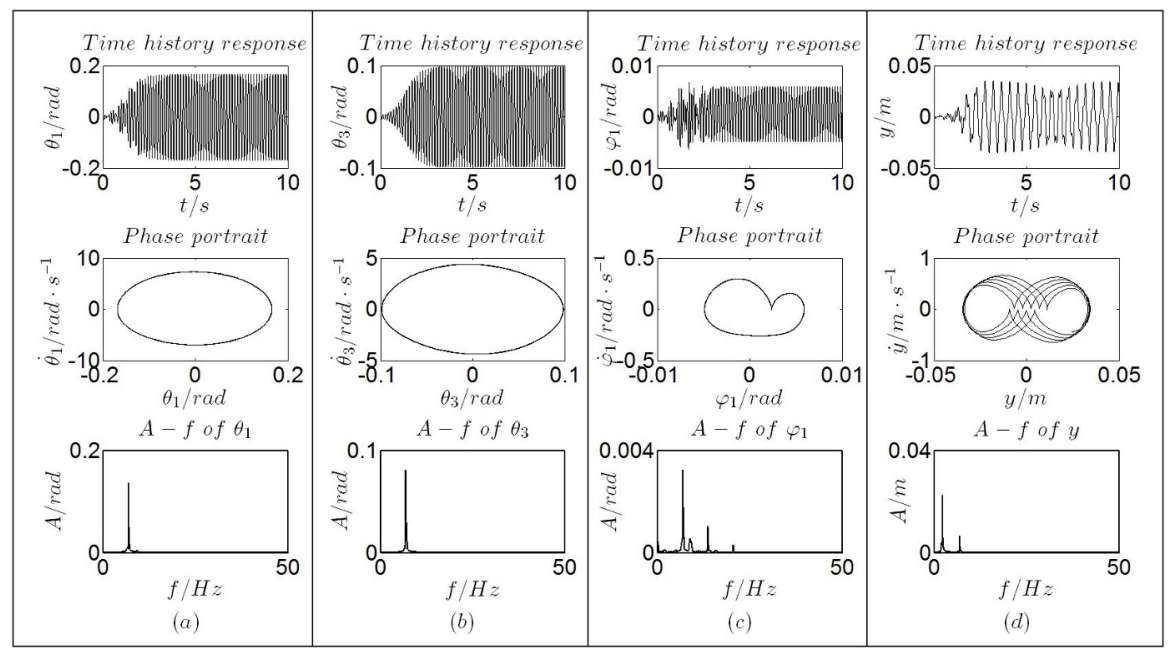

Fig. 3. Dynamic response of shimmy.

\section{Numerical analysis and simulation}

\subsection{Dynamic response of electric vehicle shimmy}

Based on the mathematical model of FIMID EV front wheels shimmy and the parameters of laboratory prototype vehicle and tire model, numerical calculation and simulation can be used to study the problem of shimmy. The dynamic response of EV shimmy are shown in Figure 3, where $(a),(b),(c)$ and $(d)$ are the dynamic response of front-left wheel shimmy, front-left axis lateral swing, pitman arm swing and vehicle lateral sway respectively, and the time history response, phase portrait and amplitude-frequency characteristic of each DOF are shown. According to nonlinear self-excited vibration theory that under the initial excitation the solution of each variable in nonlinear system is periodic oscillation, thus the front wheels of EV vibrate around its kingpin continuously, and the other DOFs of the system also produce corresponding sustained vibration.

Due to the symmetry of vehicle structure, the front-left wheel and front-right wheel in vehicle have same characteristic of dynamic response. Thus only the dynamic response of front-left shimmy and front-left axis lateral swing are given in Figure 3, and the following conclusions can be made. 
- Vehicle enter steady state shimmy rapidly after its front-left wheel was excited, namely the front wheels shimmy around the kingpin, the front wheel axes swing around the $\mathrm{x}$ axis, the pitman arm swings around its axis wire, and the vehicle sway breadthwise along y axis.

- The phase portrait of front-left wheel shimmy, front-left wheel axis lateral swing and pitman arm swing are all limit cycle, but the phase portrait of vehicle lateral sway is complex, which is similar to a kind of aperiodic vibration.

- The frequencies of front wheel shimmy, front wheel axis lateral swing and pitman arm swing are same, the frequency of vehicle lateral sway is different from others.

\subsection{Comparison between the Shimmy of electric vehicle and fuel-engined vehicle}

Because in-wheel motor is mounted in the wheel of EV, the weight of wheel and the moment of inertia of wheel in all directions in EV is increased in comparison with the FEV, and when vehicle are powered by power batteries instead of engines, its weight and COG are changed. The change of the weight of wheel and whole vehicle must influence the performance of vehicle shimmy.

If the in-wheel motor is selected, its parameters will be known. Considering the change of the weight of EV caused by replacing the engine by power battery, the amplitude of each DOF can be obtained by numerical calculation, and the results can be used to analysis the relationship between the amplitude of each DOF and vehicle velocity in shimmy.

When shimmy occurs, the relationship between the amplitude of each DOF in EV and FEV and velocity is shown in Figure 4, where the solid line represents FEV, and the dashed line represent EV. It can be seen that only when vehicle is in the moderate velocity rang, shimmy is likely to occur, and this range is defined the velocity range of shimmy. If velocity get into this range, shimmy appears, and if drop out, shimmy disappears. Vehicle shimmy is actually the bifurcation phenomena of nonlinear system. The Hopf bifurcation is produced in the system when shimmy appears or disappears due to the change of vehicle velocity. The velocity at which the Hopf bifurcation of vehicle shimmy is produced is defined as the critical velocity. The following conclusions can be made from Figure 4.

- When vehicle velocity increases, the curve of the amplitude of the front wheel axis lateral swing varies with velocity present saddle shape whose local minimum is found in the middle velocity region and local maximum is found in the high velocity region and the low velocity region, and the amplitudes of other DOFs increase first and then decreases.

- Compared with FEV, the velocity range of EV shimmy moves to the low velocity region, that is to say, the EV is more prone to shimmy in the low velocity region.

- The amplitude of each DOF of EV is greater than that of FEV.

- Compared with FEV, the amplitude of lateral sway of EV increases obviously in the low velocity region.

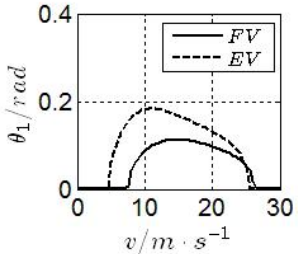

(a)

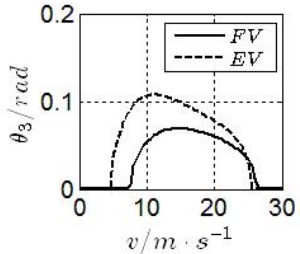

(b)

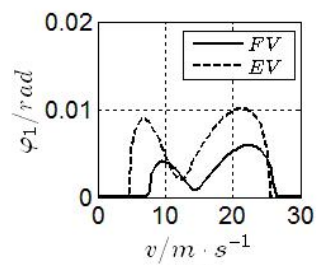

(c)

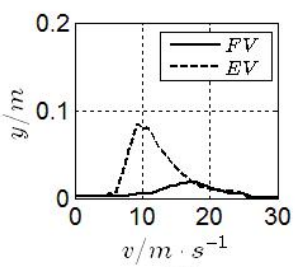

(d)

Fig. 4. Influence of vehicle velocity on the amplitude of each degrees of freedom of electric vehicle shimmy. 


\subsection{Influence of vehicle structural parameters on shimmy}

According to the characteristics of FIMID EV's structure, the influence of parameters, such as the caster angle, the inclination angle of front suspensions and the position of EV's COG, on vehicle shimmy are studied, and the amplitude of each DOF varies with these parameters are shown in Figure 5, in which $(a)$ is the influence of caster angel, $(b)$ is the influence of the inclination angle of front suspensions, and $(c)$ is the influence of the position of EV's COG. Since the magnitude of amplitude of the front axes lateral swing is small, its value is magnified by ten times in figures.

\subsubsection{Influence of the caster angle}

The caster angle $\gamma$ is the angle in the longitudinal plane of vehicle between the axis of kingpin and the line perpendicular to the ground, which can produce stable aligning torque. It can be seen from Figure 5(a) that when $\gamma$ increases, the amplitude of each DOF in the system also increases, while the amplitude of front wheels shimmy and front wheel axes lateral swing increases obviously.

\subsubsection{Influence of the inclination angle of front suspension}

The inclination angle of the front suspensions $\theta_{\mathrm{s}}$, which is marked in Figure 1, is the angle between the centre line of the front suspension spring and the horizontal plane. It can be seen from Figure 5(b) that the amplitudes of front wheel shimmy, front wheel axes lateral swing and pitman arm swing are not obvious when $\theta_{\mathrm{s}}$ increases, but the amplitude of vehicle lateral sway obviously decrease.

\subsubsection{Influence of the longitudinal position of EV's COG}

The ratio of $L_{f}$ and $L_{r}$ can be used to indicate the longitudinal position of the COG of vehicle, and the ratio of $L_{f}$ and $L_{r}$ increase when the COG moves backward. As can be seen from Figure 5(c), the amplitude of each DOF decreases when the COG of vehicle moves backward, and vice versa.

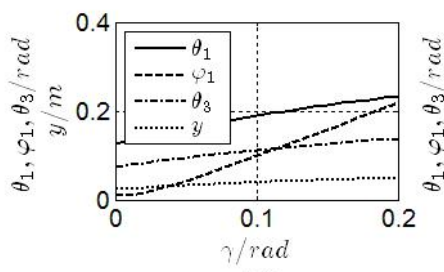

(a)

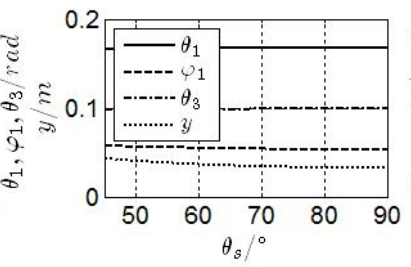

(b)

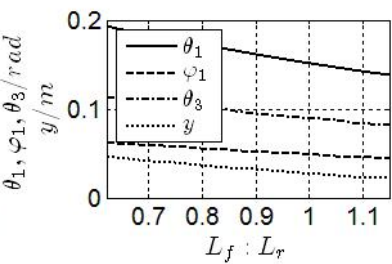

(c)

Fig. 5. Influence of vehicle structural parameters on the amplitude of shimmy.

\section{Conclusion}

Based on the 6 DOFs model of FIMID EV front wheel shimmy, the shimmy problems of $\mathrm{EV}$ are studied by numerical analysis and simulation, which is compared with that of FEV. The influence of parameters on the shimmy are studied. The following conclusions can be obtained:

- The amplitude of each DOF in EV is larger than that of FEV when vehicle shimmy, and EV is more prone to shimmy in the low velocity zone. 
- The caster angle has a great influence on vehicle shimmy, and the amplitude of each DOF increases obviously when the caster angle is increased.

- The inclination angle of front suspensions have a little influence on the amplitude of vehicle lateral sway, but its influences on other DOFs are not obvious.

- The longitudinal position of EV's COG has a great influence on the amplitude of shimmy, and the amplitude of each DOF decreases when the COG moves backward, and vice versa.

\section{Acknowledgments}

The author(s) received the supported by the National Natural Science Foundation of China (grant number 51375086 and grant number 51605087) and the Natural Science Foundation of Jiangsu Province, China (grant number BK20160671).

\section{References}

1. Eto, R., Sakata, K., \& Yamakawa, J. 2018. Driving force distribution based on tyre energy for independent wheel-drive vehicle on rough ground. Journal of Terramechanics, 76, 29-38.

2. Zhou, H., Jia, F., \& Jing, H. et al. 2018. Coordinated longitudinal and lateral motion control for four wheel independent motor-drive electric vehicle. IEEE Transactions on Vehicular Technology, 67(5), 3782-3790.

3. Jiang, X., Chen, L., \& Xu, X. et al. 2018. Analysis and optimization of energy efficiency for an electric vehicle with four independent drive in-wheel motors. Advances in Mechanical Engineering, 10(3).

4. Becker, G., Fromm, H., \& Maruhn, H. 1931. Schwingungen in Automobillenkungen, Shimmy.

5. Pacejka, H. B. 1965. Analysis of the shimmy phenomenon. Proceedings of the Institution of Mechanical Engineers Automobile Division, 180(1965), 251-268.

6. Ran, S., Besselink, I. J. M., \& Nijmeijer, H. 2014. Application of nonlinear tyre models to analyse shimmy. Vehicle System Dynamics, 52(sup1), 387-404.

7. Li, S., \& Lin, Y. (2006). Study on the bifurcation character of steering wheel selfexcited shimmy of motor vehicle. Vehicle System Dynamics, 44(sup1), 115-128.

8. Lu, J. W., Xin, J. Y., Vakakis, A. F., \& Bergman, L. A. 2015. Influences of system parameters on dynamic behavior of the vehicle shimmy system with clearance in steering linkage. Journal of Vibration \& Control, 21(2), 359-370. 